\title{
Gène H19 de la souris, empreinte parentale et syndrome de Beckwith-Wiedemann
}

L'empreinte ou sceau parental, se traduisant par une expression différente d'un même gène selon qu'il est hérité du père ou de la mère, a déjà fait l'objet de plusieurs revues dans $\mathrm{m} / \mathrm{s}$ ( $\mathrm{\circ}^{\circ} 6$ vol. 5 , p. 480-8 et $n^{\circ} 3$, vol. 7, p. 247-54). Jusqu'à présent, chez la souris, deux gènes avaient été identifiés: celui du récepteur de l'insulin-like growth factor 2 (Igf2R), sur le chromosome 17 , d'expression uniquement maternelle, et celui de l'Igf2 lui-même, d'expression uniquement paternelle, sur la partie distale du chromosome 7. Une équipe de Princeton (NJ, USA) vient d'en décrire un troisième, également sur le 7 [1]. Le gène dit H19 de la souris code pour un des ARN les plus abondants de l'embryon; exprimé dès le stade blastomère, il s'accumule dans les tissus embryonnaires, puis est réprimé après la naissance sauf dans le muscle strié. Sa séquence [2] ne révèle pas de longue phase ouverte, mais en offre environ 35 courtes*. Le produit actif du gène pourrait donc être un $\mathrm{ARN}$ et non une protéine. Comme H19 est situé dans une zone du génome soumise pour une part à empreinte parentale, Bartolomei et al.[1] ont cherché à distinguer l'expression des deux allèles paren-

\footnotetext{
- Une équi pe française dirigée par Serge Leibovitch (IGR, Villejuif) a isolé à partir d'une banque musculaire un clone d'ADNc très similaire à H19... mais codant pour une protéine contre laquelle un anticorps a été obtenu (communication personnelle). Le caractère non codant du messager H19 pourrait donc être
}

taux. Ils ont eu recours à une technique connue en génétique de la souris, le croisement entre Mus musculus et Mus spretus, dont les ADN comptent de nombreuses différences. Par la méthode de protection vis-à-vis de la RNAse, ils ont pu reconnaître l'origine paternelle ou maternelle de l'ARN H19 des descendants. L'ARN du foie montrait sans équivoque que seul l'allèle maternel est exprimé. Pour éviter un biais dû à l'emploi de ces souches particulières, des croisements entre d'autres sous-espèces de souris, ainsi que des ARN provenant d'autres tissus, notamment du muscle, furent examinés, avec le même résultat. Une dernière confirmation fut obtenue avec l'étude sur plusieurs générations : l'expression spécifique d'allèle est remise en cause à chaque génération. Par exemple, une femelle F1 ( $M$ domesticus $\times M$ spretus), qui exprime H19 domesticus venant de sa mère, et dont l'allèle spretus paternel reste muet, est croisée avec un mâle $M$ domesticus : les descendants qui ont hérité de leur mère l'allèle spretus l'expriment exclusivement.

Il existe donc deux gènes sur le chromosome 7 subissant l'empreinte parentale, exprimant l'un l'allèle paternel et l'autre l'allèle maternel. Or des études de recombinaison [1] montrent que ces deux gènes, Igf2 et H19, sont étroitement liés, ainsi qu'au gène de l'insuline, qui, lui, échappe au sceau parental. Les deux gènes qui lui sont soumis ont sans doute une importance biologique : les hétérozygotes ayant hérité un gène Igf2 inactif de leur père sont de taille réduite [3]. Quant à H19, son importance dans la régulation est attestée par le fait que son introduction sous forme de transgène conduit à la mort de l'embryon entre 14 et 16 jours [1]. On ne peut donc impunément modifier le nombre de gènes H19. Enfin, l'influence de la partie distale du chromosome 7 vient d'être encore soulignée par les expériences de Ferguson-Smith et al. (Cambridge et Oxford, GB), montrant que des embryons chimériques porteurs d'une duplication paternelle de cette région ont une croissance très accélérée [4]. En l'absence de données précises, on ne peut que spéculer sur des applications éventuelles à l'homme. H19 et Igf2 ont tous deux été assignés au bras court du chromosome 11, en $11 \mathrm{p} 15.5$, au voisinage immédiat du gène de l'insuline. Or cette région semble soumise à empreinte parentale. Le groupe de Claudine Junien à Paris [5] a montré qu'une maladie qui peut s'accompagner de tumeur de Wilms et qui comporte une croissance foetale excessive, appelée syndrome de Beckwith-Wiedemann, est associée à une disomie uniparentale : les deux portions correspondantes du bras court du 11 proviennent uniquement du père, et même, dans plusieurs cas, du même chromosome paternel. Cette disomie n'inclut pas la totalité du chromosome 11 et notamment pas son bras long. On ne sait actuellement rien du rôle, chez ces malades, de $\mathrm{H} 19$ et de Igf2. Si la régulation est la même chez l'homme et la souris, une origine uniquement paternelle impliquerait une production accrue d'Igf2, au moins pendant la période embryonnaire - et pas de production de H19 du tout. Dans un éditorial de Nature [6], M. Little et al. discutent longuement la possibilité qu'un excès d'Igf2 soit à l'origine d'une croissance trop rapide, et suggèrent qu'Igf2 pourrait 
être le gène responsable du syndrome de Beckwith-Wiedemann. Il est tout aussi plausible d'attribuer un rôle à H19, qui pourrait être un modérateur de la croissance ; on pourrait, surtout, accorder de l'intérêt au déséquilibre du fonctionnement de ces deux gènes, qui pourrait être à l'origine d'anomalies, même si, à l'évidence, elles ne seraient pas les mêmes que celles de la souris. Reste à prouver que, chez l'homme, ces gènes sont soumis à empreinte parentale. S'il semble difficile de détecter avec certitude un doublement de l'expression de l'Igf2, il devrait être possible de mettre en évidence une absence de production de H19. Ce gène, chez la souris, reste exprimé dans le muscle adulte. Chez l'homme, il se peut que se maintienne une expression résiduelle dans d'autres tissus plus facilement accessibles. Bien entendu, même si ces spéculations s'avéraient exactes, elles n'apporteraient pas la preuve du mécanisme d'action de la disomie uniparentale dans la genèse du syndrome. Ce n'en serait pas moins un bon point de départ pour sa compréhension.

J.C. D.

1. Bartolomei MS, Zemel S, Tilghman SM. Parental imprinting of the mouse H19 gene. Nature 1991; 351: 153-5.

2. Brannan CI, Dees EC, Ingram RS, Tilghman SM. The product of the H19 gene may function as an RNA. Mol Cell Biol 1990 ; 10 : 28-36.

3. DeChiara TM, Robertson EJ, Ef stratiadis A. Parental imprinting of the mouse Insulin-like growth factor II gene. Cell 1991; 64: 849-59.

4. Ferguson-Smith AC, Cattanach BM, Barton SC, Beechey CV, Surani MA. Embrylogical and molecular investigations of parental imprinting on mouse chromosome 7. Nature 1991; 351: 667-70.

5. Henry I, Bonaiti-Pellić, Chehensse V, et al. Uniparental paternal disomy in a genetic cancer-predisposing syndrome. Nature $1991 ; 351: 665-7$.

6. Little M, Van Heyningen V, Hastie N. Dads and disomy and disease. Nature 1991 ; 351: 609-10

$m / s \quad n^{\circ} 7$, col. 7, septembre 91

\section{OIT BRÈVE}

L'acide ursodésoxycholique dans la cirrhose biliaire primitive : affirmation d'un acquis thérapeutique. La plupart des médicaments essayés dans le traitement de la cirrhose biliaire primitive* l'ont été pour leur action immunosuppressive. Le plus efficace semble être la ciclosporine. En 1987, l'acide ursodésoxycholique fut proposé comme une nouvelle approche thérapeutique [1], fondée sur l'hypothèse que les lésions hépatiques y étaient le résultat de l'accumulation intracellulaire d'acides biliaires potentiellement toxiques, et que l'acide ursodésoxycholique acide biliaire hydrophile dépourvu de cytotoxicité in vitro et in vivo chez l'homme - pourrait réduire, en administration prolongée, la réabsorption des acides biliaires endogènes et modifier la composition de leur pool. Des résultats d'études non contrôlées et des résultats préliminaires d'une étude contrôlée publiés par R.E. Poupon, R. Poupon et al. (Inserm U. 21, Villejuif, et service d'hépatologie, hôpital Saint-Antoine, Paris) semblaient confirmer l'hypothèse initiale [2]. La récente publication des résultats complets de cette étude prospective contrôlée et multicentrique (19 centres cliniques en France, un au Canada) - sur 146 cas de cirrhose biliaire primitive - démontre que l'acide ursodésoxycholique est efficace, sur une période de trois ans, au triple plan clinique, biologique et anatomique [3]. Cliniquement, la proportion de malades ayant, à trois ans, une maladie évolutive ne diminue de manière significative que dans le groupe traité ; le prurit y est significativement (bien que de quelques $\%$ seulement) moins fréquent, et son intensité, si gênante pour les malades, est notée comme très atténuée. Biologiquement, les signes de cholestase diminuent de façon marquante dans le groupe traité, notamment le taux moyen des phosphatases alcalines (divisé par 2), et celui de la $\gamma$-glutamyl transférase (divisé par 4), de même que diminue significativement le titre des anticorps anti-mitochondries et le score de ris- que (de la Mayo Clinic), index prédictif de survie au cours de la maladie. Histologiquement, le score mesurant la sévérité des lésions ne s'améliore vraiment que dans le groupe traité, l'amélioration concernant la rareté des canaux biliaires, l'inflamation portale et péri-portale à cellules mononucléées, le degré de prolifération ductulaire, l'intensité de la cholestase, de la nécrose hépatocytaire, mais non celle de la fibrose. Les effets secondaires de l'acide ursodésoxycholique se sont limités, chez un seul malade, à l'aggravation du prurit, obligeant à l'arrêt du traitement. Ce travail montre clairement que l'acide ursodésoxycholique est efficace, et dépourvu d'effet secondaire, dans la cirrhose biliaire primitive. Il s'impose donc, actuellement, comme le traitement de choix de cette affection, au moins dans les conditions de cette étude : c'est-à-dire cirrhoses biliaires primitives symptomatiques mais de sévérité modérée ou moyenne, l'efficacité de l'acide biliaire étant probablement corrélée positivement au niveau résiduel de la circulation entéro-hépatique des sels biliaires. Le mécanisme d'action de l'acide ursodésoxycholique pourrait donc impliquer une inhibition de l'absorption iléale d'acides biliaires endogènes hépatotoxiques, et peutêtre aussi un effet sur le système immunitaire hépatique : il diminue l'expression des antigènes HLA de classe I dans les cellules des canaux biliaires et pourrait ainsi réduire la cible désignée des cellules $\mathrm{T}$ cytotoxiques.

- La cirrhose biliaire primitive est une affection probablement auto-immune caractérisée par une destruction des petites voies biliaires interlobulaires avec cholestase intrahépatique et altérations hépatocytaires secondaires.

[1. Poupon R, et al. Lancet $1987 ; 1$ 834-6.]

[2. Poupon RE, et al. Hepatol 1990 ; 11: 16-21.]

[3. Poupon RE, et al. N Engl J Med $1991 ; 324$ : 1548-54.] 